\title{
Débat scolaire : les enjeux anthropologiques d'une didactisation
}

\section{Michel Tozzi}

\section{(2) OpenEdition \\ Journals}

Édition électronique

URL : http://journals.openedition.org/trema/598

DOI : 10.4000/trema.598

ISSN : 2107-0997

Éditeur

Faculté d'Éducation de l'université de Montpellier

Édition imprimée

Date de publication : 1 mai 2004

Pagination : 49 - 57

ISSN : 1167-315X

Référence électronique

Michel Tozzi, « Débat scolaire : les enjeux anthropologiques d'une didactisation », Tréma [En ligne], 23 | 2004, mis en ligne le 30 septembre 2010, consulté le 19 avril 2019. URL : http://

journals.openedition.org/trema/598; DOI : 10.4000/trema.598

Ce document a été généré automatiquement le 19 avril 2019

Trema 


\section{Débat scolaire : les enjeux anthropologiques d'une didactisation}

Michel Tozzi

Etat des lieux 
1 Le débat est à l'ordre du jour dans le système éducatif français :

2 - Dans la vie scolaire (ex : la formation des élèves délégués).

3 - Dans la vie de classe : on encourage dans le primaire la mise en place de « conseils » de type Freinet ou «pédagogie institutionnelle »; on a institutionnalisé

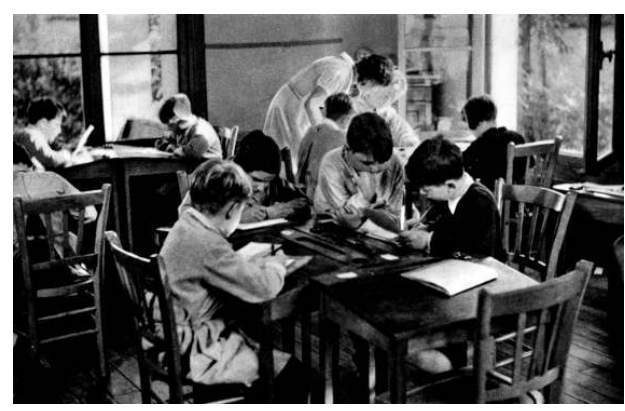
une heure de vie de classe dans le secondaire, avec des « débats de régulation ».

4 Dans l'enseignement des disciplines : en français on demande des « débats d'interprétation » oraux sur des textes à partir de passages qui "résistent» dès la maternelle, on vise à développer des capacités argumentatives par des discussions dans le cadre d'une didactique de l'oral en constitution ; en éducation civique (rebaptisée « vivre ensemble »), on a rendu obligatoire en cycle 2 et 3 du primaire un temps de débat par semaine ; en sciences économiques et sociales on débat sur des «questions socialement vives »; en mathématiques on organise à partir de «problèmes ouverts " des «débats scientifiques "; en sciences expérimentales on confronte par le débat des hypothèses à partir d'énigmes sur des phénomènes naturels (cf. la démarche de « la main à la pâte »).

5 - En ECJS au lycée (qui n'est pas une discipline mais un "enseignement»), on a explicitement pour objectif la «méthodologie du débat argumenté». Dans l'expérimentation de la philosophie en lycée professionnel, la discussion l'emporte sur le cours magistral ou la dissertation. Les innovations font une large part à la discussion : on voit émerger dans le système éducatif des «discussions à visée philosophique » au primaire et en collège etc.

6 On peut interpréter cette récurrence, cette insistance comme un symptôme. A un niveau explicite, ce succès pourrait s'expliquer parce que le débat est à la rencontre de finalités jugées actuellement décisives pour le système éducatif. Il est à la confluence :

- de la maîtrise orale de la langue, dont il est un des principaux genres, et qui est un indicateur fondamental des difficultés et de l'échec scolaires ;

- de l'éducation à la civilité et à la citoyenneté, jugées prioritaires à cause de la montée des « incivilités », d'une part parce qu'il met en jeu une éthique communicationnelle de la personne, d'autre part parce qu'il relie politiquement son apprentissage dans l'école de la République à celui de la participation démocratique d'un citoyen critique dans l'espace public ;

- de la co - construction des savoirs dans la classe, qui constitue une « communauté de recherche » à partir de problèmes, d'énigmes, de questions, selon le paradigme socio constructiviste des didactiques disciplinaires, où le conflit socio - cognitif permet d'apprendre en modifiant ses représentations.

7 Il est objectif d'apprentissage (" apprendre à débattre »), par exemple en didactique de l'oral en français, pour maîtriser un genre de l'oral comme « genre scolaire » (au sens de SCHNEUWLY); en éducation civique, pour savoir intervenir dans «l'espace public scolaire » de la classe et l'établissement. Mais aussi moyen d'apprentissage disciplinaire de savoirs et de savoir faire, et de socialisation démocratique pour «vivre ensemble en apprenant ». 
Ce faisant il tente d'articuler, c'est son aspect « symptomatique », ce qui fait crise à l'école (et dans la société) :

- le rapport à la loi, par une relation plus coopérative au pouvoir, acceptant le bien fondé des règles de l'échange, qui échappe tant à l'autoritarisme rejeté qu'à un laxisme anomique ;

- le rapport au savoir, par une relation signifiante, non dogmatique et socialisée à la connaissance, plus conforme à la conception épistémologique moderne du rapport à la vérité ;

- la quête de sens, en redonnant une signification aux apprentissages scolaires (ex : le débat en mathématiques), ou à sa propre vie (discussion à visée philosophique).

9 Il tente de réduire le déficit de sens sociétal et scolaire : celui du lien social et politique dans une société individualiste qui a perdu l'utopie des "grands récits» (LYIOTARD), le repère d'une transcendance à la fois antérieure, extérieure et supérieure aux individus, et dans laquelle la prétention à la validité passe désormais, y compris en sciences, par l'espace de la discussion pour construire un monde commun.

10 Si l'école tente aujourd'hui de didactiser le débat, d'en faire un objet culturel à transmettre et s'approprier, si une société juge que le débat doit être appris par les enfants parce qu'il est nécessaire au monde de demain, c'est qu'il y a des enjeux anthropologiques en question pour la condition humaine et son avenir.

\section{Le sens anthropologique du débat scolaire}

11 - Discuter en classe, c'est un certain rapport à l'autre, sur le mode de l'interaction. Interaction entre personnes, implicitement ou explicitement prises au niveau psychologique dans les affects projectifs de sympathie ou de rejet de leur inconscient, dans des stratégies plus ou moins volontaires de domination ou de séduction, où la "face " (GOFFMAN) de chacun est exposée et négociée, et qui engage au niveau éthique des valeurs, par exemple le respect du «visage » (LEVINAS) de l'autre, ou la tolérance à l'altérité des personnalités et des visions du monde. Education de soi, éducation de « soi comme un autre » (RICCEUR), éducation à l'autre, à son « étrange étrangeté » (FREUD).

12 - C'est même une interaction plurielle, qui concerne plusieurs personnes à la fois, et non une relation duelle, un "dialogue " à deux, même si chacun s'adresse souvent à une personne précise. La discussion se fait en groupe, et la parole tourne, multipliant pour chacun les interlocuteurs potentiels, amenant plusieurs personnes à intervenir. Discuter est une modalité psychosociologique et sociale du "vivre ensemble" selon un certain mode relationnel, interactif et groupal, le travail en groupe.

13 - Cette interaction est de nature verbale, à base d'échanges en langue naturelle (pour les élèves le français), plus globalement langagier, incluant dans cette transaction orale le sujet de l'énonciation en situation pragmatique, avec le para verbal de l'intonation par exemple, ou le corporel des gestes et mimiques (le non segmentaire comme disent les linguistes). Type d'interlocution qui engage les règles de la langue, mais plus généralement celles de la communication, telles que dégagées par exemple dans l'analyse conversationnelle, avec tours de parole et « texte polygéré ».

14 - C'est une interaction à visée psychologique ou psychosociologique s'il s'agit d'un débat de régulation, cognitive s'il s'agit d'un apprentissage disciplinaire, parce que la discussion a toujours un objet qui fait tiers et médiation dans la relation purement interpersonnelle : un point à l'ordre du jour, un thème, une question, un problème, un concept. En ce sens 
elle est centrée, et doit en principe échapper à la pensée purement associative de la "conversation", parce qu'elle traite d'un sujet. Le groupe qui discute est une sorte « d'intellectuel collectif » qui se donne un objet de travail, et le « discutant » adopte une « posture » à la fois langagière, psychologique, psychosociologique et sociale, affective et cognitive, orientée vers une "production » : l'apaisement dans la régulation de conflits, une décision dans des discussions à visée démocratique, l'approfondissement d'une question ou la résolution d'un problème intellectuel dans une discussion d'échange ou d'appropriation conceptuelle.

- Communication interactive partagée, impliquant la réciprocité de l'échange, où il est souhaitable que chacun tienne compte de ce qui a été dit précédemment, réagisse à l'urgence d'une altérité incarnée qui surprend et interpelle, et où l'horizon d'attente de cette communauté discursive est la participation de chacun au travail collectif de cette intersubjectivité.

16 - Communication en contexte, ici institutionnel, celui de la classe ou de la vie scolaire, où chacun, en fonction de son statut, joue un rôle, tient une fonction, exerce un «métier d'élève » ou une " profession » d'enseignant ou d'éducateur, dans le cadre d'une relation éducative, pédagogique ou didactique.

17 - Avec de forts enjeux. Au niveau individuel, influencer l'autre, par des effets psycho et socio - affectifs, rhétoriques ou conceptuels de persuasion ou de conviction (« Dire c'est faire »), avec un rapport de force qui peut être implicite ou explicite : débattre, ce peut être tenter de battre, lutter contre, vouloir avoir raison (de l'autre), jusqu'à tuer avec des mots «assassins». Mais ce peut être aussi chercher avec, s'enrichir mutuellement, apprendre au contact des autres. Enjeu collectif de trouver ensemble une réponse à une question, une solution à un problème, de progresser dans une réflexion, s'approprier des connaissances, aboutir à une décision mûrie ...

18 - Echange civilisé, car réglé et régulé. Une discussion scolaire a des conditions d'existence et de possibilité : elle a un début et une fin, un cadre spatio - temporel, une gestalt comme "genre scolaire " ... Pour discuter, il faut se comprendre, et donc d'abord s'entendre physiologiquement, ne pas parler plusieurs en même temps. D'où à cause du nombre un tour de parole, le pouvoir et le devoir de se taire pour que fonctionne le droit d'expression de chacun. "Laisser place » à l'autre pour admettre sa présence, le droit d'expression de sa parole, de la singularité de sa différence, tolérer en silence provisoire une divergence est formateur pour l'égocentrisme de chacun, élève et maître. C'est un apprentissage de la co - existence et de la reconnaissance. Travail sur soi pour différer, surseoir, retenir ses pulsions, maîtriser la toute puissance. Quiconque sent cette impatience, ce bouillonnement, cette exaspération à vouloir intervenir, couper, prendre de la place, prendre la place, sa place, (le) faire taire, devenir le centre du groupe, et se contraint un instant, quelques minutes au silence, sait combien l'assomption de cette frustration est porteuse de victoire sur le soi archaïque qui veut plier l'autre et le monde à son désir.

19 La discussion est discipline, cela s'apprend, et c'est un rôle socialisateur de l'école. Elle suspend la violence physique, commence quand cessent les coups, continue tant qu'ils ne concluent pas, prévient même les coups ou les fait cesser dès qu'elle s'instaure. Il y a là un processus d'hominisation, d'humanitude (JAQUARD), dans cette irruption de la parole qui rompt la spontanéité non médiatisée qui va d'un trait de l'émotion ressentie au coup qui part. La parole s'interpose entre des protagonistes antagonistes. Elle fait pause parce qu'elle immobilise le corps en minimisant son emballement global et pulsionnel, et 
canalise vers le visage et la bouche son expressivité. Elle amortit la radicalité du choc en métamorphosant le combattant en débatteur, et en sollicitant le cerveau cortical de la rationalité. Cet arrêt, cet interdit de la violence, parce qu'il est un inter - dit (la parole est un dire inter, entre humains) est en même temps et par là même une autorisation : je peux parler parce que les autres se taisent. A chacun une place, toute sa place, rien que sa place, parce que chacun aura, s'il en manifeste la volonté, son moment. Respect et équité, il y a dans l'apprentissage de la discussion une portée éthique par le respect de la personne, et démocratique par le partage du pouvoir.

Certes, dans une perspective « réaliste ", on peut penser que, comme « la diplomatie est la continuation de la guerre par d'autres moyens " (CLAUZEWITZ), la parole est la continuation de la violence par le langage, y compris sous les formes masquées de la «distinction» (BOURDIEU), violence "symbolique» de ceux qui sont en position socioculturelle « haute » (GOFFMAN). Car la parole, et la prise de parole sont un pouvoir, celui de l'influence. On connaitt les injures verbales, qui sont de la violence par les mots, et l'on sait que certaines phrases ouvrent des plaies narcissiques (quand on dit par exemple à un élève : "Tu es nul »). Mais une injure, une dépréciation, un ordre ne sont pas du débat, puisqu'ils sortent de l'espace du "discutable». La discussion commence quand cesse la violence langagière de l'affect, la parole agressive, quand il y a place pour le silence, l'alternance, quand on peut terminer sa phrase. L'argument est une violence apprivoisée, civilisée par la raison. Ce n'est plus la force ou la ruse qui fait autorité dans une discussion, mais l'argument.

Si la discussion est un mode d'expression sur les conflits, et parfois de résolution, c'est parce que la parole fait tiers qui réinclut. La verbalisation des affects retisse chez le sujet une parole intérieure, la parole refait lien entre les protagonistes, l'autre est réentendu dans sa logique et son intérêt, une négociation devient possible quand chacun a repris pied chez l'autre.

Mais qu'il s'agisse de réguler un groupe, de prendre une décision ou de confronter de idées, une discussion suppose toujours, pour ne pas dysfonctionner :

- une régulation des processus interpersonnels et de dynamique de groupe. Faute d'accompagnement des affects, la confrontation des idées peut à tout moment dériver en confrontation de personnes, le conflit sociocognitif en conflit socio - affectif, où ça dispute mais ne discute point.

- un réglage des procédures, c'est - à - dire des modes explicites de fonctionnement organisé : on adopte un ordre du jour, ou on ne dérive pas du sujet abordé, on demande la parole, on ne parle pas trop longtemps, on ne coupe pas ni ne se moque de celui qui parle etc.

Il existe dans des sociétés africaines un bâton de parole pour réglementer la palabre. Mais certaines pratiques qu'a inventées l'humanité ont affiné ce réglage à cause de leur spécificité. Ce n'est pas un hasard si le débat politique sur l'agora, le procès juridique, le débat scientifique et le dialogue philosophique naissent ensemble dans la Grèce antique. Car la discussion commence où s'arrête le dogme, vérité absolue, définitive, extérieure et supérieure, autorité transcendante (Dieu, le Pape, le Coran, le Roi, le Duce, le « père du peuple »...), dont l'ascendant s'impose donc ne se discute pas, et en dehors duquel on est religieusement hétérodoxe, politiquement dissident ou intellectuellement indécrottable. Discuter présuppose d'ouvrir le champ du «discutable», donc du questionnable et de l'interrogé, du problématique et du vraisemblable (ARISTOTE, PERELMAN), du possible et du probable, donc de l'incertain. C'est entrer dans une culture de la question et en finir 
avec l'évidence de la réponse, c'est oser interroger la tradition et la transmission, c'est subvertir les fondations et déconstruire, soupçonner.

Peut-on encore fonder quand on relativise ? Ou on tombe dans le perpectivisme sceptique (PYRRHON, NIETZSCHE), ou on pense que la raison peut être le moyen d'obtenir l'accord des esprits par la discussion (APPLE, HABERMAS). C'est ce rationalisme discussionnel que nous avançons, parce que, sans préjuger de la connaissance des « choses en soi » (KANT), il permet de donner un sens, dans l'espace de l'intersubjectivité humaine, à la co construction du savoir scientifique et à la discussion tant démocratique qu'à visée philosophique.

\section{Quelle didactisation?}

25 - Le propre de la discussion démocratique, en tant qu' "idéal régulateur », c'est que chacun et tous ont un droit égal à la parole, sans prévalence d'un chef, du plus fort, du plus fortuné, du plus ancien, voire du plus sage. Les Grecs recouraient au tirage au sort, instituant une parité des hommes libres au sein même d'une asymétrie des connaissances et des compétences. Chacun compte pour un au moment du vote, et le minoritaire, qui peut devenir majoritaire, voit garantir son droit d'expression. Cet idéal, même s'il est souvent démenti par les faits, repose sur des valeurs de liberté et d'égalité d'expression, de pluralité des opinions, dans un « espace public » qui en délimite et garantit le cadre. Et même si la démocratie assure la distinction vie publique / vie privée comme sécurité de droits, la discussion, en ce qu'elle est exercice d'une liberté et d'une égalité des citoyens, a toujours un caractère de publicité, parce qu'elle s'appuie sur le jugement de la raison éclairée par «l'instruction publique». Elle traduit l'engagement d'un homme et d'un citoyen dans une position rationnelle et civique à tenir en public.

C'est ce sens de l'engagement citoyen qu'il faut préparer au sein de «l'espace public scolaire ». Pour organiser cet espace public de discussion, la démocratie s'est donnée des pratiques de référence: des fonctions nécessaires, comme président ou secrétaire de séance, rapporteur, ou des instances de discussion : groupes de travail, conseil ... Et aussi des procédures et des outils, comme un ordre du jour, une liste d'inscription, un temps d'intervention, un compte rendu...Ce sont ces démarches qui peuvent être didactisées en classe (cf. le conseil coopératif de la pédagogie institutionnelle) et dans la vie scolaire (en donnant un contenu concret aux droits des élèves).

- Mais la discussion n'est pas seulement une méthode politique de délibération en vue de décider du bien commun d'un groupe, c'est aussi épistémologiquement la façon dont se construit historiquement le savoir scientifique. Contrairement à l'opinion commune selon laquelle un savoir scientifique n'est plus discutable puisqu'il a été reconnu comme «vrai », l'épistémologie contemporaine, par exemple chez K. POPPER, avance qu'est scientifique ce dont on pourrait montrer que c'est faux, c'est - à - dire ce qui est «falsifiable». Le savoir scientifique, c'est précisément ce qui est discuté, ce qui fait problème dans une communauté internationale de chercheurs. Ce savoir est co construit, dans un mélange inextricable, comme le montre la sociologie de la connaissance, de mobiles personnels, d'intérêts financiers, de moyens techniques, de positions et de stratégies institutionnelles, d'administration de la preuve. Il est historique, évolutif, donc relatif, ce qui ne veut pas dire arbitraire, car il faut en permanence dans le débat soumettre la validité de ses résultats à la critique de ses pairs, 
dont le consensus est nécessaire pour reconnaître le caractère scientifique de la proposition. Si la pratique sociale de référence d'élaboration du savoir scientifique est la co - construction par le débat, cette approche d'un savoir socialisé doit être didactisée à l'école, à travers la démarche du débat scientifique en classe, notamment à partir de conflits sociocognitifs.

- Mais le savoir scientifique n'est pas la seule forme culturelle de rationalité. Si la discussion «à visée philosophique» se développe aujourd'hui en France, c'est parce qu'elle développe un rapport au savoir non dogmatique sur des questions qui font sens pour les élèves. ARISTOTE distinguait la discussion « éristique » et la discussion « heuristique ». La première est de type agonistique: l'autre est un adversaire à (con-) vaincre. C'est la logique sophistique d'une argumentation pour ou contre, sans synthèse possible ni même déplacement de la question. Il y en a un de trop, et c'est l'autre, qu'il s'agit d'amener sur sa position ou de disqualifier ; à moins qu'il ne s'agisse de se concilier par la démagogie le nombre, pour emporter la décision. Discuter, c'est alors un rapport de force ou / et de séduction, un pari et un défi où il s'agit de gagner. Ce qui est en jeu, c'est d'exercer sur autrui et le groupe le pouvoir du langage, sans l'ordonner au souci du rapport au savoir et à la vérité. Le sophiste peut ainsi soutenir indifféremment un point de vue et l'inverse, avec le même challenge, rallier l'auditoire. Si l'on pense que l'école doit adapter à la dureté et aux ruses de la réalité sociale, elle didactisera alors les pratiques sociales, prises comme références, de l'avocat, du publicitaire, de l'homme politique : apprendre à argumenter, c'est - à - dire à faire partager son point de vue...Mais l'école doit - elle seulement s'adapter, c'est - à - dire prendre comme «pratiques sociales de référence » (MARTINAND) les habitus sociétaux, par exemple le débat médiatique? Ce qui semble justifié pour un diplôme professionnel (ex : BTS « force de vente ») doit être critiquement interrogé en formation générale commune quant à sa portée éducative (quelles valeurs promouvoir pour quel homme dans quelle société de demain ?).

Car la discussion peut être "heuristique». L'autre devient un partenaire dans une " communauté de recherche» (LIPMAN). Je ne lutte plus contre, je cherche avec. Une objection n'est plus une agression contre ma personne, mais un cadeau intellectuel pour approfondir ma pensée. Toute thèse prend statut épistémologique d'hypothèse soumise au groupe pour éprouver (soumettre à la preuve) sa validité.. Et je suis demandeur de critique, dont je vois méthodologiquement le bien fondé. L'autre n'est pas de trop, mais nécessaire dans son altérité pour me décentrer, car mes objections à mes idées se heurtent aux limites des cadres de ma pensée. La différence est une opportunité, un KAIROS pour m'affronter à moi - même par une confrontation à autrui. Sa singularité m'interpelle : comment peut-il penser le contraire puisque je crois avoir raison? Je peux respecter sa personne, mais sa pensée divergente me scandalise intellectuellement: il faut que j'en discute avec lui, pour savoir qui de nous deux a raison, et si ce que je crois est vraiment fondé! Et s'il s'avère que je suis réflexivement trop « léger », je ne serais pas vaincu, mais heureux d'avoir progressé. On n'est jamais vaincu par la raison dès qu'on adhère à ses raisons. La discussion heuristique implique non seulement une éthique relationnelle (avoir le souci de ce que pense l'autre), mais de la pensée (se rendre à la raison), parce qu'elle a pour tâche la recherche de la vérité, l'amour du savoir, au sein d'une communauté d'esprits rationnels visant l'universalité. De ce point de vue, l'école doit promouvoir des valeurs, celles du respect d'autrui, de la recherche de la vérité, et apprendre à résister à la sophistique, la doxologie, la démagogie. Elle ne doit donc pas didactiser n'importe quelle pratique sociétale. C'est pourquoi nous plaidons, notamment 
en français, pour une didactique de l'argumentation ancrée sur un oral réflexif (BUCHETON), qui considère qu'une thèse (le "pour" ou le "contre»), n'a anthropologiquement de sens que si elle n'est qu'une des réponses à une question qui est un problème parce qu'il y a des enjeux forts pour l'humanité. Ce qui devrait écarter toute rhétorique purement formelle, tout développement de compétences coupé de leur dimension axiologique, tout sujet sans enjeu, du type " pour ou contre le sac à dos ou le cartable classique » (sic dans un manuel de cycle 3), et articuler tout apprentissage de l'argumentation à celui de la problématisation sur des thèmes humainement porteurs, qui font sens pour les élèves.

\section{Conclusion}

L'apparition du débat et sa valorisation dans l'histoire humaine nous semble avoir anthropologiquement une triple dimension :

- politique, en ce qu'il instaure entre les hommes l'organisation démocratique d'un espace public de délibération en vue d'un bien commun, sur la base de principes de liberté, d'égalité et d'argumentation (rapport au pouvoir partagé) ;

- épistémologique, en ce qu'il est un mode incontournable de co - construction du savoir scientifique, et une modalité d'élaboration de la pensée philosophique (rapport au savoir et à la vérité) ;

- éthique, en ce qu'il est ce passage d'un rapport de pouvoir fondé sur la force à un monde intersubjectif commun, réglé a minima par la civilité des conditions de possibilité d'une discussion (respect du 'visage ' de l'autre) , au mieux par la coopération constructive dans une recherche commune de vérité (morale de la pensée).

C'est dans cette perspective que peut prendre sens éducatif sa didactisation scolaire.

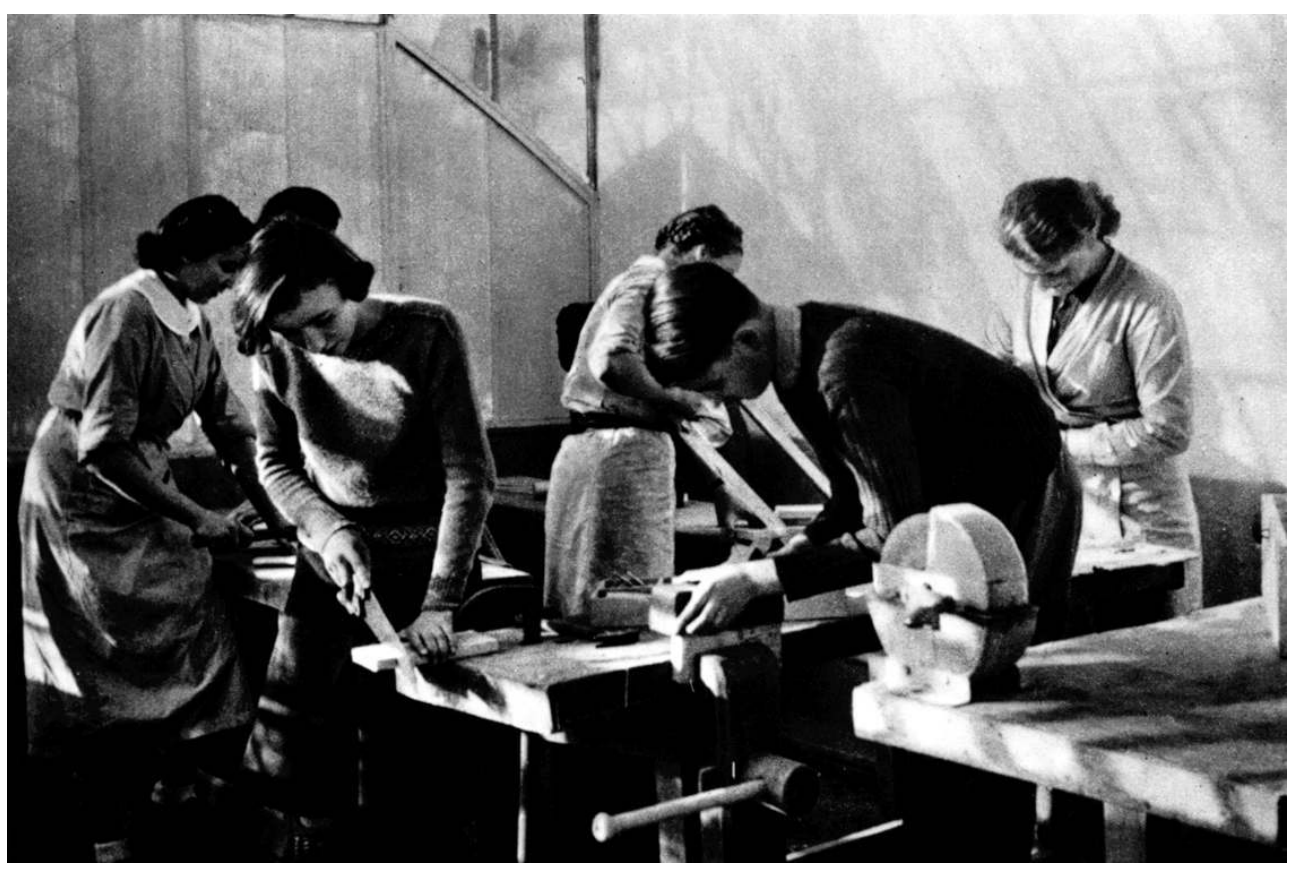

Les activités manuelles au service de la culture générale : découpage des cartes géographie, maquette de maisons, etc.

Image (légèrement réduite) tirée de : L'enfant d'âge scolaire / Barrier P. Paris : Horizon de France, 1937. - (Le visage de l'enfance). P. 304 


\section{BIBLIOGRAPHIE}

Débattre en classe, Cahiers pédagogiques, fév.2002, nº 401 .

TOZZI M., 1999, L'oral argumentatif en philosophie, Crdp Montpellier.

TOZZI M., COORD. :

- 2001, L'éveil de la pensée reflexive à l'école primaire, Cndp - Hachette.

- 2002, La discussion philosophique à l'école primaire, Crdp Montpellier.

- 2002, Nouvelles pratiques philosophiques en classe, Crdp Bretagne.

\section{RÉSUMÉS}

L'apparition du débat dans l'humanité a des enjeux anthropologiques forts : politique (partage démocratique du pouvoir), épistémologique (démarche socialisée de co - construction du savoir), éthique (respect d'autrui et morale de la pensée). Mis à l'ordre du jour de notre système éducatif, c'est à partir de ces enjeux qu'il doit être didactisé, comme objectif et moyen d'apprentissage : apprendre la discussion, apprendre par la discussion, apprendre à vivre ensemble par la discussion.

The apparition of the debate in human history contains some strong anthropological stakes : political stakes (democratic division of power); epistemological stakes (socialized procedure to built a common knowledge); ethical stakes (respect of others and caring thinking). Put on the educational agenda, it must be "didactised", as objective as well as means of learning: learning to discuss, learning through discussion, learning to live together through discussion.

\section{INDEX}

Mots-clés : débat, débat démocratique, débat scientifique, didactisation, discussion à visée philosophique, enjeu anthropologique

\section{AUTEUR}

\section{MICHEL TOZZI}

Professeur d'université en sciences de l'éducation à Montpellier III, Directeur du CERFEE - IRSA 